
\title{
BReserch S Suare \\ The Expression of Ptch1 and Ptch2 in the Stenotic Tissue of Congenital Ureteropelvic Junction Obstruction in Children
}

\section{Yanfang Yang}

Henan University of Technology

wenwen han ( $\square$ HAN_WEN_WEN@YEAH.NET)

beijing children's hospital

\section{Weiping Zhang}

Capital Medical University

Ning Sun

Capital Medical University

Research article

Keywords: ureteropelvic junction obstruction; immunofluorescence; Ptch1; Ptch2

Posted Date: May 21st, 2020

DOI: https://doi.org/10.21203/rs.2.21792/v2

License: (c) (i) This work is licensed under a Creative Commons Attribution 4.0 International License.

Read Full License 


\section{Abstract}

Background: Ptch1 and Ptch2 are expressed in tubular epithelium and stromal cells adjacent to the UPJ. They mediate inhibition of Smoothened, a transmembrane protein expressed on the cell surface. If the pathway is disturbed, UPJOcan occur. This aim study aimed to determine the expression of Ptch1 (P1) and Ptch2 (P2) in stenotic segments in children with congenital ureteropelvic junction obstruction (UPJO) compared with normal control subjects.

Methods: Stenotic segments of ureter tissues were obtained from 20 UPJO patients.UPJO caused by other pathogenies, such as vessel and ureteral polyps, were excluded. The control ureter specimens were obtained from 10 patients with Wilm's tumor, and the tissues were confirmed histologically to be unaffected. Immunofluorescence, western blot and real-time PCR were used to investigate the expression of P1 and P2. Statistical methods were used to find the differences between the two groups

Results: P1 and P2 were identified in the cytoplasm of smooth muscle in two groups through immunohistochemistry. However, there were no statistical differences between the two groups in P1 and $P 2$ with immunohistochemistry ( $P=0.31$ and $P=0.3$, respectively). There were also no statistical differences with western blot $(P=0.75$ and $P=0.9$, respectively) and real-time $P C R(P=0.52$ and $P=0.45$, respectively). However, with the immunofluorescence it was found that red-stained $\mathrm{P} 1$ were diffused in the controls group, but were mainly located in the intracellular perinuclear compartment of smooth muscle cells in UPJO.

Conclusions: The expression of P1 and P2 between the two groups had no statistical significant. P1 were mainly located in the intracellular perinuclear compartment of smooth muscle cells in UPJO. The P1 pathway might be disturbed by the abnormal distribution rather than the quantity, which might be one probable pathogenesis of UPJO.

\section{Background}

Ureteropelvic junction obstruction (UPJO) is the most common form of congenital urinary tract obstruction (1). Most cases of UPJO are caused by intrinsic narrowing of the ureter at the renal pelvis, although the underlying pathogenic mechanisms are not defined exactly (2). Sonic hedgehog (Shh) signaling pathway was found to be involved in kidney and ureter embryonic development $(3,4)$. Several researchers havefound that the pathway was disturbed inanimal models and UPJO children (5).

Shh and its receptor Patched are found in the tubular epithelium and stromal cells of the UPJ (6). Shh binds to Ptch and relieves Smoothened, which is a transmembrane Ptch-mediated inhibition protein expressed on the cell surface (5). Full-length Gli3 proteins translocate to the nucleus after binding, acting as transcriptional activators (7). If the pathway is disturbed, UPJO might occur.

There are two Ptch homologs in mammals, Ptch1 (P1) and Ptch2 (P2), both of which bind to Shh ligands with similar affinity (8). Despite various investigations, the Ptch regulation in UPJO children remains 
unclear, and whether there is variation is yet to be proved (9). The expression of P1 and P2 have been compared in hydronephrotic models and contiguous segments in the same patients' ureters in previous studies, but the same parts of human ureteropelvic junction segments have not been compared $(5,10)$.

In this study, the expression of P1 and P2 in stenotic segments in UPJO children compared with normal control subjects was investigated. The normal control specimens were obtained from ureteropelvic junction segments of Wilm's tumor patients.Immunohistochemistry, western blot and real-time PCR were usedto find the possible pathogenic mechanisms in UPJO.

\section{Methods}

\section{Patients and control samples}

The study was based on information retrieved between June 2018 and June 2019 and was approved by the Ethics Committee (2019-k-368). UPJO was diagnosed based on some examinations, such as ultrasound, intravenous pyelography and diuretic renogram. Surgical indications followed EUA guidelines. Intrinsic stenosis was confirmed during the operation. UPJO caused by other pathogenies, such as vessel and ureteral polyp, were excluded. Altogether, there were 20 stenotic ureter specimens from children with UPJOand 10 control ureter specimens from children with Wilm's tumor. The children with Wilm's tumor were not given pre-operation radiotherapy or chemotherapy and the specimens were confirmed by histology to be unaffected. The UPJO specimens were obtained strictly. First, after cutting off the specimen, the "stenotic" portion was split longitudinally and the obviously narrow ureter found. Second, the inconspicuous narrowing was removed at both ends. Lastly, each specimen was cut into at least three pieces lengthwise and immediately stored $a t-80^{\circ} \mathrm{C}$ preparing for immunofluorescence, WB and PCR respectively. In the control samples, 5-millimeter long pieces of ureteropelvic junction were cut off and the same process applied as for the specimens with in UPJO.

\section{Immunofluorescence of P1 and P2}

Formalin-fixed and paraffin-embedded tissues were stained by immunohistochemistry to observe the expression of P1 and P2. Immunofluorescence was performed on $4 \mu \mathrm{m}$ paraffin sections. Dako serumfree protein block was used to block, primary and secondary incubation buffers. Whole-mount immunofluorescence was performed as usual. The antibodies used were: P1 (primary antibodies: goat, lot: ab39266; fluorescent secondary antibodies: CY3 labeled rabbit anti-goat lgG) and P2 (primary antibodies: rabbit, lot: ab238338; fluorescent secondary antibodies: 488 Labeled goat anti- rabbit lgG) (Abcam company, 1:100). Each patient's tissue was divided into three pieces, and one piece from each was evaluated by two investigators with a confocal microscope (Nikon Ci-S, Tokyo, Japan). This was then used to split up the light spectrum and make a quantitative analysis of fluorescence intensity using Inform software (PerkinElmer). Five photomicrographs ( $\times 200)$ were randomly selected to count the fluorescence intensity, and the means of fluorescence intensity were calculated as the ratio of integral optical density. 


\section{Western blot of Ptch1 and Ptch2}

Pre-cooled RIPA protein extraction reagents (50 mMTris-HCl(pH 7.4), $150 \mathrm{mM} \mathrm{NaCl}, 1 \% \mathrm{np}-40,0.1 \%$ SDS) were added to a protease inhibitor cocktail (Roche) (phosphorylated protein needed to be added to phosphatase inhibitor at the same time) (according to the volume ratio of 50:1). The tissue was cut into pieces, and $50 \mathrm{mg}$ of tissue was added to $500 \mathrm{ul}$ lysate. The tissue was centrifuged at $13000 \mathrm{rpm}$ for $20 \mathrm{~min}$. The supernatant was removed and the tissue stored at -80 degrees for Western blot. The separation gel was prepared according to the molecular weight of the target protein. Electrophoresis, incubation of primary and secondary antibodies and film exposure were then performed. The primary antibodies were P1 (goat, 1:300) and P2 (rabbit, 1:1000), and the secondary antibodies were goat anti rabbit IgG and goat anti mous IgG (1:10000). Band density was quantified by digital densitometry using the Gel Image system ver.4.00 (Tanon, China). Band intensity was normalized to that of $\beta$-actin.

\section{RNA isolation and RT-PCR}

The ABI 7500 fluorescence quantitative PCR instrument was used, and $\triangle \Delta C t$ method was used for relative quantitative analysis of the data. Quantitative real-time PCR was accomplished with SYBR Premix Ex Taq (TaKaRa) on LightCycler-GmbH D-68298 (Roche Molecular Biochemicals) under the following conditions: $95^{\circ} \mathrm{C}$ for $10 \mathrm{~s}, 45$ cycles of $95^{\circ} \mathrm{C}$ for $5 \mathrm{~s}, 58^{\circ} \mathrm{C}$ for $20 \mathrm{~s} ; 65^{\circ} \mathrm{C}$ for $15 \mathrm{~s}$. A dissociation procedure was performed to generate a melting curve for confirmation of amplification specificity.Primer sequences were as follows: P1 forward, 5'-CGCTCTGGAGCAGATTTCCA-3', reverse, 5'CTCGTCCTCCAACTTCCACC3; P2 forward 5'- GCCGCCAGAGGTGATACAG-3', reverse, 5'GTGTGTCTGATGAGGGGGTG-3'. Beta actin 5'-ACAGAGCCTCGCCTTTGCC-3', reverse, 5'GATATCATCATCCATGGTGAGCTGG-3'. The relative changes in expression levels of P1 and P2 were normalised against the levels of GAPDH gene expression in each sample ( $\triangle \Delta \mathrm{Ct}$ method). They were carried out in triplicate for each sample and primer.

\section{Statistical analysis}

Statistical software SPSS 22.0 was used to analyse data. All normally distributed data were presented as mean \pm SD. Significance of differences was evaluated using a two-sample $t$ test. $P$ value $<0.05$ was considered to be statistically significant.

\section{Results}

The UPJO patients ranged from 6 months to 12.5 years old, with a mean age of 3.3 years. The control group ranged from 1.2 months to 7 years old, with a mean age of 3.2 years. No statistical differences were found between the two groups with regard to the age of patients $(P=0.28)$.

\section{Immunofluorescence}

P1 was specifically stained red and P2 was green, while the nucleuses of smooth muscle cells were stained blue. In the UPJO group, expressions of P1 and P2 were found in stenotic segments (Figure 1A 
and 1C). In the control group, expressions of $\mathrm{P} 1$ and $\mathrm{P} 2$ were detected in ureteropelvic junction segments (Figure 1B and 1D). There were no statistical differences between the two groups in P1 and P2. The results of the immunofluorescence and $P$ values between the two groups are summarised in Table 1. Redstained P1 were equally diffused in the control group (Fig.1B), but were mainly located in the intracellular perinuclear compartment of smooth muscle cells in UPJO.

Table 1: Results and $P$ values of the immunofluorescence, western blot and mRNA

\begin{tabular}{lcccc}
\hline & & UPJO & Control & P \\
\hline IM & P1 & $2.37 \pm 0.96$ & $2.11 \pm 1.5$ & 0.31 \\
& P2 & $2.62 \pm 1.95$ & $2.93 \pm 2.8$ & 0.30 \\
WB & P1 & $0.58 \pm 0.08$ & $0.57 \pm 0.09$ & 0.75 \\
& P2 & $0.44 \pm 0.15$ & $0.44 \pm 0.11$ & 0.90 \\
PCR & P1 & $3.3 \pm 1.3$ & $2.6 \pm 1.9$ & 0.52 \\
& P2 & $5.3 \pm 2.2$ & $5.3 \pm 1.5$ & 0.45 \\
\hline
\end{tabular}

\section{Western blot}

The western blot method was used to examine the expression level of P1 and P2 in the UPJO and control groups (Figure 2). The level of protein expression was calculated, followed by calculation of the relative intensity through standardisation using the expression of $\beta$-actin as the internal control. P1 was $0.58 \pm 0.08$ in UPJO and $0.57 \pm 0.09$ in the control; P2 was $0.44 \pm 0.15$ in UPJO and $0.44 \pm 0.11$ in the control. The expression of $\mathrm{P} 1$ and $\mathrm{P} 2$ between the two groups was not statistically significant (Pख0.05). The results of the western blot and $P$ values between the two groups are summarised in Table 1 .

\section{Real-time PCR}

Amplification and melting curves were drawn according to the mRNA fluorescence values and cycle numbers of P1 and P2, using $\beta$-actin as the internal control (Figure 3). Each sample was repeated five times in real-time PCR test and averaged as the Ct value. The $2^{-\triangle \Delta \mathrm{ct}}$ relative quantitative method was used for mRNA expression analysis by ABI 7500 fluorescence quantitative PCR instrument. The difference in mRNA expression of P1 and P2 in the two groups was notstatistically significant $(P<0.05)$ (Table1).

\section{Discussion}

There has been much research on molecule mechanisms underlying congenital intrinsic UPJO, but it is still poorly understood (11). Research has demonstrated that dysregulated Shh-Ptch-Gli3 dependent mechanisms could cause murine and human intrinsic UPJO (5). Shh signaling consists of ligand membranes of Ptch receptors, downstream target genes and pathway regulation proteins (4). During renal development, the Shh and its receptor Ptch are expressed in stromal cells and tubular epithelium. 
Shh binds to Ptch and relieves a transmembrane protein expressed on the cell surface (6). In the presence of Shh, GLI proteins (Gli3) are translocated to the nucleus, acting as transcriptional activators (12).

It has previously been shown that Shh signaling control cellular differentiation during ureter formation. The defection in urothelial differentiation can cause UPJO in mice (13). Previous studies have found increased Shh and decreased Gli3 expression in children with UPJO (4). Shh might increase because the downstream target is disturbed. Since the Shh receptor is Ptch, the signal is blocked in this phase, so the next downstream target Gli3 is decreased. What happens in the intermediate passage? The results are inconclusive. Recent research has focused on the Ptch structure $(8,11)$, but it had still not proved that the expression of Ptch (P1 and P2) is abnormal in UPJO. Gupta et al. found that the expression of P1 in hydronephrotic mice model was high compared to normal (10). His research was based on artificial mutant mice, not on real UPJO patients. In Sheybani's research, P1 increased in a single patient out of eight (5), but the control tissue was a contiguous UPJO segment, so these results do not describe a real pathogenic mechanism underlying congenital UPJO.

In this study, 20 intrinsic UPJO tissues and 10 ureters from children with Wilms tumor were obtained. Even so, the results were not very good, demonstrating that P1 and P2 were not changed in the quantity of UPJO through immunofluorescence, western blot and PCR. All the results were negative and an impasse was reached in the research. Previous studies found that P1 was removed from cilia after binding of the Shh ligand to P1, allowing Smoothened (A G-coupled transmembrane protein) to enter cilia and become activated. If $\mathrm{P} 1$ is not removed, it prevents Smoothened's localisation to cilia and disrupts the Gli transcription activator/Gli3 repressor (Gli3R) balance (7). According to this molecular biology, the P1's distribution may be abnormal in the UPJO patients if this pathway has been disturbed. Examining the immunofluorescence of P1 again, it was found that red-stained P1 were diffused in the control group (Fig.1B), but were mainly located in the intracellular perinuclear compartment of smooth muscle cells in UPJO. Based on previous findings, it was inferred that P1's quantity was unchanged, but the movement was restricted in UPJO. Thus, the whole Shh pathway was disturbed. This is merely a hypothesis that we inferred from our thisstudy, and further study is needed to confirm this.

P2 in the Shh signaling pathway remains ambiguous. Research has shown that the two homologs have overlapping functions, but they cannot compensate for the loss of P1 activity (14). Studies have found that P2 does not play a functional role in regulating Shh signaling activity during kidney development, but serves as a specific marker of the onset of UPJO (5). In some studies, P2 increased in UPJO (5), but in this research, $\mathrm{P} 2$ was not changed and no regularities of distribution could be found. The rules of specimen collection of uretero-pelvic-junction in the UPJO and control groups were strictly followed. In other studies, the control group did not use the human uretero-pelvic junction.

\section{Limitations}

Only two factors of P1 and P2 were assayed. The upstream signal Shh and downstream target factors, such as Smoothened and Gli3, were not tested in this research, because they have been verified in many 
studies $(3,4)$. The objective of this found was to prove the variation of Ptch. Although the abnormal distribution and unchanged quantity of Ptch were seen, nothing was found. Further study on the interaction between $\mathrm{P} 1$ and Smoothened will be carried out in the future to explore the pathogenesis of UPJO.

\section{Conclusions}

The expression of P1 and P2 between the two groups was not statistically different. P1 were mainly located in the intracellular perinuclear compartment of smooth muscle cells in UPJO. The P1 pathway might be disturbed by the abnormal distribution of $\mathrm{P} 1$ rather than the quantity, which might be one probable pathogenesis of UPJO.

\section{Abbreviations}

P1 Ptch1

P2 Ptch2

UPJO ureteropelvic junction obstruction

\section{Declarations}

\section{Authors' contributions.}

YFY and WWH conceived of the study and performed the experiments and wrote the manuscript. WWH was done the acquisition of data, or analysis and interpretation of date. WPZ participated in the design of the study and provided technical advice. NS was involved in the design of the study, in discussions and reviewed the manuscript. All authors read and agreed to the final manuscript.

\section{Funding.}

A grant from the Beijing Municipal Administration of Hospitals'Youth Programme (QML20181210) supported this study. The funding body did not have any additional role in the study design, data collection and analysis, and manuscript preparation.

\section{Availability of data and materials.}

The datasets generated and analyzed during the current study are available from the corresponding author on reasonable request.Ethics approval and consent to participate: The experimental protocol was approved by the Ethics Committee of Beijing Children's Hospital, Capital Medical University. We obtained all the written consents from the participants or their parents in the study. Consent for publication: Not applicable.Competing interests: The authors declare that they have no competing interests. 
Acknowledgements.

Not applicable.

\section{References}

1. Carlström M. Hydronephrosis and risk of later development of hypertension. Acta Paediatr. 2019;108(1):50-7.

2. Krajewski W, Wojciechowska J, Dembowski J, Zdrojowy R, Szydełko T. Hydronephrosis in the course of ureteropelvic junction obstruction: An underestimated problem? Current opinions on the pathogenesis, diagnosis and treatment. Adv Clin Exp Med. 2017;26(5):857-64.

3. Hu MC, Mo R, Bhella S, Wilson CW, Chuang PT, Hui CC, et al. GLI3-dependent transcriptional repression of Gli1,Gli2 and kidney patterning genes disrupts renal morphogenesis. Development. 2006;133(3): 569-78.

4. Chen H, Ji HY, Yang Y. The Expression of Gli3 and Teashirt3 in the Stenotic Tissue of Congenital Pelvi-Ureteric Junction Obstruction in Children. Int J Med Sci. 2016;13(6):412-17.

5. Sheybani-Deloui S, Chi L, Staite MV, Cain JE, Nieman BJ, Henkelman RM, et al. Activated HedgehogGLI Signaling Causes Congenital Ureteropelvic Junction Obstruction. J Am Soc Nephrol. 2018;29(2):532-44.

6. Rohatgi R, Milenkovic L, Scott MP. Patched1 regulates hedgehog signaling at the primary cilium. Science. 2007;317(5836):372-6.

7. Blake J, Hu D, Cain JE, Rosenblum ND. Urogenital development in Pallister-Hall syndrome is disrupted in a cell-lineage-specific manner by constitutive expression of GLI3 repressor. Hum Mol Genet. 2016;25: 437-47.

8. Gong X, Qian H, Cao P, Zhao X, Zhou Q, Lei J, et al. Structural basis for the recognition of Sonic Hedgehog by human Patched1. Science. 2018;361(6402). pii: eaas8935.

9. Fleet AJ, Hamel PA. The protein-specific activities of the transmembrane modules of Ptch1 and Ptch2 are determined by their adjacent protein domains. J Biol Chem. 2018;293(43):16583-95.

10. Gupta DP, Hwang JW, Cho ES, Kim W, Song CH, Chai OH. Constitutive Activation of Smoothened in the Renal Collecting Ducts Leads to Renal Hypoplasia, Hydronephrosis, and Hydroureter. Cells Tissues Organs. 2017;204(1):38-48.

11. Qi X, Schmiege P, Coutavas E, Wang J, Li X. Structures of human Patched and its complex with native palmitoylated sonic hedgehog. Nature. 2018;560(7716):128-32.

12. Nieuwenhuis E, Hui CC. Hedgehog signaling and congenital malformations. Clin Genet. 2005;67:193-208.

13. Yan J, Zhang L, Xu J, Sultana N, Hu J, Cai X, et al. Smad4 regulates ureteral smooth muscle cell differentiation during mouse embryogenesis. PLoS One. 2014; 9(8):e104503. 
14. Zhulyn O, Nieuwenhuis E, Liu YC, Angers S, Hui C-C. Ptch2 shares overlapping functions with Ptch1 in Smo regulation and limb development. Dev Biol.2015;397:191-202.

\section{Figures}
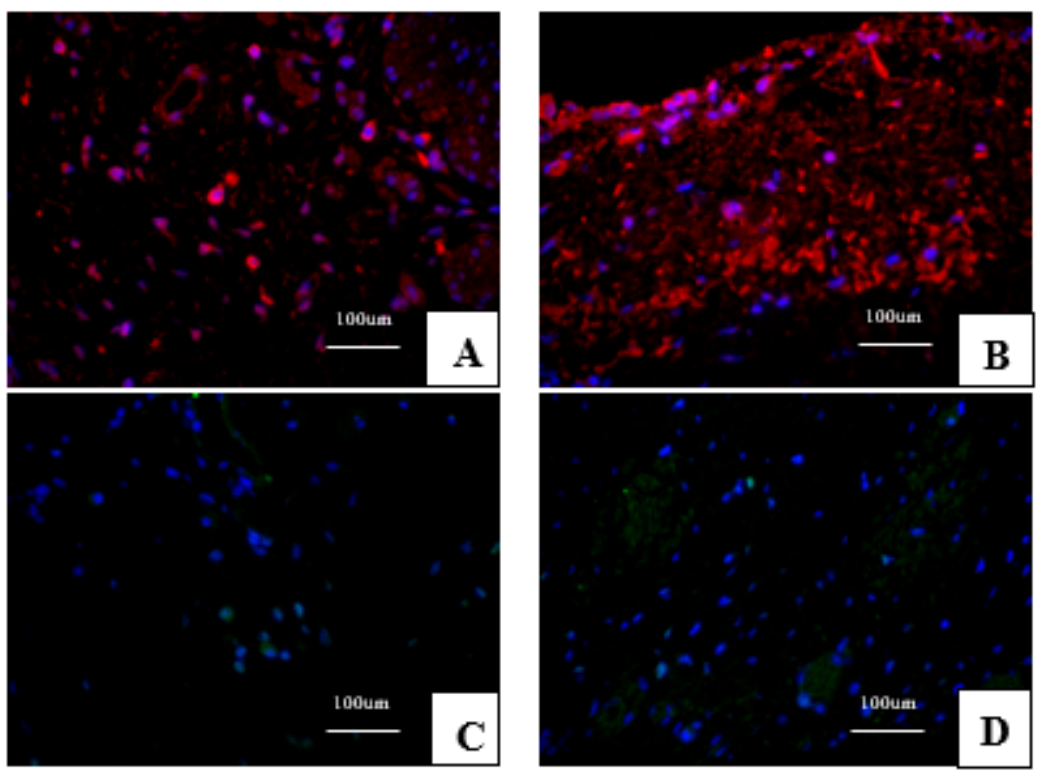

\section{Figure 1}

Ptch immunohistochemistry stain (×400). A: P1 was specifically stained red in the stroma in UPJ0 group. P1 were mainly located in the intracellular perinuclear compartment of smooth muscle cells (stained blue). B: P1 was stained red and diffused in the stroma in normal control group. C D: P2 was specifically stained green in the stroma in two groups.

\begin{tabular}{|l|l|l|l|l|l|l|l|l|l|}
\hline $\mathrm{X} 1$ & $\mathrm{x} 2$ & $\mathrm{x} 3$ & $\mathrm{X} 4$ & $\mathrm{x} 5$ & $\mathrm{x} 6$ & $\mathrm{x} 7$ & $\mathrm{x} 8$ & $\mathrm{~N} 1$ & $\mathrm{~N} 2$ \\
\hline
\end{tabular}

Ptch1

Ptch2

Actin

\section{Figure 2}

We choose 10 representative samples to show here. Western blot analysis of P1 and P2 protein expression in UPJO patients and in controls. $\mathrm{X}$ indicates UPJO group and $\mathrm{N}$ control group. The protein expression of P1 and P2 in UPJO tissue was not statistics significance comparing with the control group. 

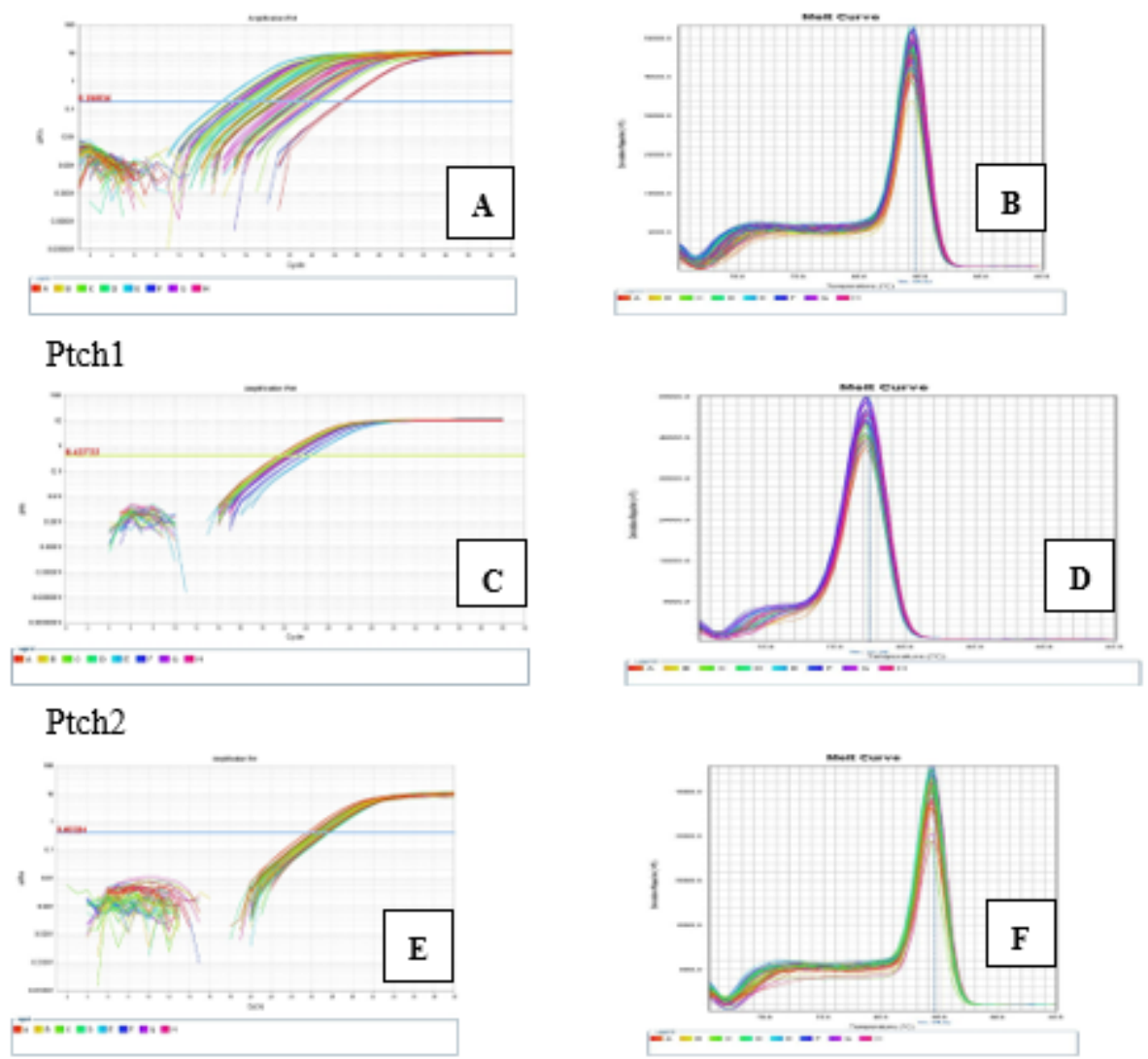

Figure 3

Real-time PCR analysis of P1 and P2 mRNA expression in UPJO and control groups. A B: Amplification and melting curves for Beta actin; C D: Amplification and melting curves for P1 mRNA expression; E F: Amplification and melting curves for P2 mRNA expression. 